\title{
BORDERSCAPING: REPENSAR FRONTEIRAS E MIGRAÇ̃̃ES NO MEDITERRÂNEO ATRAVÉS DAS AGÊNCIAS POLÍTICAS NA FRONTEIRA ÍTALO-TUNISIANA ${ }^{1}$
}

\author{
CHIARA BRAMBILLA \\ Professora da Universidade de Bergamo (Itália) \\ chiara.brambilla@unibg.it
}

\section{Para além da linha}

O Mediterrâneo revelou-se, nos últimos anos, um espaço crucial para o estudo das fronteiras, não como entidades "tidas como certas", exclusivamente relacionadas com os limites territoriais externos dos Estados nacionais, mas como lugares móveis, relacionais e sujeitos a disputas de vários tipos. (Bechev et al., 2010). Nessa perspectiva, o Mediterrâneo, como escreveu Braudel (1987, p. 9), "é uma boa oportunidade para apresentar uma 'outra' forma de aproximar-se da história”. Trata-se de uma ocasião que se origina no caráter de "espaço-movimento" do Mediterrâneo, no qual Braudel insiste em sua exploração histórica, descrevendo o grande mar entre as terras habitadas por culturas, poderes e relações migratórias, onde a alegada clareza dos limites são desafiadas pelas diferentes raízes coexistentes, pela multiplicidade de pertencimentos e vozes que coexistem na confusão de cada definição abstrata e estática (Chambers, 2008).

Desta forma, o Mediterrâneo dialoga com algumas questões que se encontram no centro da corrente de reflexão crítica sobre as fronteiras que, em nível internacional, tem se caracterizado nos últimos anos por uma crescente atenção à urgência de revisitar os seus próprios instrumentos conceituais e metodológicos a fim de compreender a natureza mutável dos processos de bordering ${ }^{2}$.

1 Originalmente publicado em italiano em Illuminazioni (ISSN: 2037-609X), n. 38, outubro-dezembro 2016. Tradução de Clarice Campani Langer e Cláudia Mendonça Scheeren (Dra. em Letras, professora adjunta da UFRGS). Revisão de João Carlos Córdova Frasson e Adriana Dorfman (Dra. em Geografia, professora adjunta da UFRGS).

2 Sobre o desenvolvimento de novas perspectivas críticas para o estudo das fronteiras, entre outros, ver: Hess et al. (2010); Parker et al. (2012); Rumford (2010); Wilson et al. (2012). 
Embora a virada processual (de border para bordering) no estudo das fronteiras como processos políticos, sociais e culturais complexos - que são produzidos por nós, mas que ao mesmo tempo contribuem para a produção de nossas identidades individuais e coletivas (Brambilla, 2010) - tenha estimulado importantes passos em frente na reflexão sobre as fronteiras, as lentes analíticas desta perspectiva revelaram-se insuficientes para compreender plenamente as muitas implicações dos contextos históricos, políticos e sociais em constante mudança nas fronteiras do sul da Europa, no Mediterrâneo e além, bem como para responder adequadamente aos cenários flutuantes e muitas vezes dramáticos do que foi rotulado como crise de refugiados e migrantes (Rajaram, 2015).

Nessa perspectiva, esta contribuição propõe algumas considerações sobre o potencial conceitual e metodológico da abordagem do borderscaping para investigar a complexidade multidimensional da conexão fronteira-migração no Mediterrâneo, não apenas do ponto de vista geopolítico, mas também epistemológico e histórico-antropológico ${ }^{3}$. A relevância da contribuição analítica oferecida pela abordagem do borderscaping não se deve apenas ao fato de que ela nos permite questionar a moderna ordem geopolítica e social centrada no Estado. As lentes analíticas do borderscaping nos permitem ir além, proporcionando uma visão "antropolítica"4, que incentiva uma melhor compreensão da complexidade multidimensional epistêmica, espacial e temporal das fronteiras, mostrando-as como um importante recurso para a construção de imaginações geopolíticas inéditas além da linha de divisão territorial entre as soberanias dos estados modernos, imaginários sociais e espaciais, imagens culturais e estratégias práticas para persegui-las (Brambilla et al., 2015, p. 2-4). Em particular, a abordagem do borderscaping favorece uma leitura construtiva e fecunda dos imaginários da fronteira mediterrânea e da conexão, passado e presente, entre as fronteiras e as migrações, focando em três tópicos: a conexão político-estética, a

3 Para uma reflexão sobre o potencial crítico do conceito de borderscape, consulte: Brambilla (2015a); dell'Agnese et al. (2015). Para um aprofundamento sobre uma abordagem interdisciplinar do borderscaping, consulte: Brambilla et al (2015).

4 Sobre o conceito de "antropolítica", consulte: Morin (2000). 
urgência de uma perspectiva genealógica, a importância de elaborar uma abordagem metodológica política e performática.

Nas páginas seguintes, faremos uma primeira pausa para esclarecer estes três tópicos para considerar, posteriormente, as orientações específicas adotadas em uma pesquisa etnográfica realizada entre 2013 e 2015 na região da fronteira ítalo/tunisiana, como exemplo de uma possível "aplicação prática" do potencial conceitual e metodológico da abordagem borderscaping para o estudo da conexão fronteiras-migrações nos cenários mediterrâneos contemporâneos ${ }^{5}$.

\section{Borderscaping, ou rumo a imaginários alternativos da fronteira mediterrânea}

A conexão entre política e estética está no centro da evolução etimológica do sufixo "-scape", revelando como a noção de borderscaping fornece uma possível chave de releitura das fronteiras, útil para liberá-las de serem reduzidas a um 'lugar visível', exclusivamente referido à imagem moderna da fronteira essencializada como uma linha divisória nos mapas. Em vez disso, o traço etimológico da noção de borderscaping destaca um duplo significado do termo, no qual o borderscape não se resolve em sua imagem estética moderna, mas expressa uma agência ${ }^{6}$ de trabalho criativo - "dar forma a", "construir" - referindo-se ao ato humano de 'modelar' um conjunto de espaços terrestres, que funcionam e evoluem para servir uma comunidade, derivando dele o caráter antropolítico do borderscaping, a sua origem e, ao mesmo tempo, o ser parte de um "projeto político do fazer" (Olwig, 2008, p. 8191). Em sua dupla declinação etimológica, o borderscape consegue assim 'manter juntas' representações e práticas por meio da conexão entre a estética e a política na qual se origina a polissemia

5 A pesquisa foi conduzida como parte do trabalho realizado em nome do Ce.R.Co. (Centro de Pesquisa em Complexidade) da Universidade de Bergamo no Projeto VII Programa-Quadro de Pesquisa e Desenvolvimento da Comissão Europeia EUBORDERSCAPES (junho de 2012-maio de 2016). Para mais informações sobre a EUBORDERSCAPES, consulte http://www.euborderscapes.eu/. Em relação à pesquisa realizada pela equipe do Ce.R.Co. como parte do Projeto acima mencionado, consulte http://www.cercounibg.it/ricerche/.

6 Agency, no original em italiano (N.T.). 
da noção (Brambilla, 2016). Trazendo à tona as interações entre as implicações políticas e estéticas da conexão fronteiras-migrações, a noção de borderscaping favorece uma 'desconstrução' crítica do imaginário geopolítico e social moderno e de suas topologias espaço-temporais, bem como da geometria euclidiana em que se baseia, (re)afirmando a ligação entre o valor multidimensional das fronteiras (com base na sua interpretação como construídas, experimentadas, interpretadas e contestadas por seres humanos) e ao seu caráter político. Ao mesmo tempo, a duplicidade etimológica do borderscaping evidencia como as configurações geográfico-políticas e sociais dos imaginários de fronteira estão intimamente ligadas à atividade estética. As linguagens estéticas regulam, orientam e reorientam os processos pelos quais as fronteiras se materializam no mundo real, bem como as maneiras pelas quais os imaginários das fronteiras são traduzidos em crenças, normas, políticas e práticas (Schimanski et al., 2013; Schimanski et al., 2017). Assim, se dá aquilo que Rancière (2011) definiu como um "diálogo sobre a divisão do sensível" entre a estética e a política, que ordena e regula o que é visível, o que pode ser dito e quem pode falar. Através da lente analítica do borderscaping, a relação entre estética e política pode ser entendida como uma tensão entre uma distribuição particular do sensível e um esforço contínuo de redistribuição progressiva e subversiva dessa ordem. Desse modo, a conexão político-estética, no centro da noção do borderscape, revela a tensão, sempre presente na fronteira, entre ser um instrumento político para o exercício da soberania do Estado moderno e sua pretendida autoridade exclusiva e a afirmação de borderscapes contra-hegemônicos, que se originam em discursos e práticas políticas e estéticas de "dissidência”, possibilitando pensar em alternativas à exclusividade estática de uma compreensão determinista do espaço-fronteira mediterrâneo ${ }^{7}$. Assumir a perspectiva analítica do borderscaping esclarece, então, a determinação conflituosa dos imaginários plurais que participam da construção do espaço-fronteira mediterrâneo (Brambilla, 2015b). Não para negar essa dimensão conflituo-

7 Para uma reflexão crítica sobre o papel dos discursos e práticas políticas e estéticas "dissensuais", assim como sobre as relações entre elas, consulte: Rancière (2007), especialmente p. 21-36. 
sa, mas para fornecer possíveis aberturas virtuosas para mostrar o potencial das fronteiras como "locais de luta", onde diferentes tensões se articulam entre formas de agências institucionais e informações (Mezzadra et al., 2014, p. 335-341). Isso evidencia que o potencial crítico das fronteiras deve ser buscado em sua dimensão de estruturas paradoxais: por um lado, são marcas de pertencimento para a definição de uma política do ser e, por outro, são lugares do tornar-se, onde novas formas de pertencimento e o tornar-se político podem ser expressos e postos em prática ${ }^{8}$. Revelando as complexas interações entre as configurações hegemônicas e contra-hegemônicas do espaço-fronteira mediterrâneo, a abordagem do borderscaping convida a um repensar crítico da ligação entre in/ visibilidade, poder e experiência vivida, indo além da retórica do "espetáculo de fronteira" no Mediterrâneo (Cuttitta, 2002; De Genova, 2013), que se articula em narrativas impostas à opinião pública através de uma comunicação bastante midiática e simplificadora, muitas vezes carente de profundidade histórica e antropológica.

Ir além da retórica do espetáculo da fronteira no Mediterrâneo significa também compreender que as fronteiras têm uma história que não pode ser esquecida pelos políticos, mas deveria ser valorizada. Dar visibilidade à marca temporal das fronteiras permite historicizar a conexão fronteiras-migrações e reafirmar a urgência de uma abordagem genealógica dessa conexão, superando a orientação a-histórica que caracteriza a maioria dos discursos e práticas sobre os desafios políticos e socioculturais contemporâneos no Mediterrâneo (Brambilla, 2014).

A abordagem do borderscaping permite-nos aprofundar a perspectiva crítica sobre as fronteiras, proporcionando uma representação 'multi-situada' dos cenários mediterrâneos não só no espaço, mas também no que diz respeito às múltiplas tensões entre os diferentes atores, tempos e métodos que participam na construção material e simbólica das fronteiras no Mediterrâneo e do Mediterrâneo como espaço-fronteira. Isso evidencia as implicações significativas que a abordagem do borderscaping gera não apenas no nível conceitual, mas também considerando seu valor

8 Sobre o conceito de borderscape e a transição de uma política do ser para uma política do tornar-se, consulte: Rajaram et al. (2007). 
como método. Para que essa abordagem metodológica seja praticada, deve-se atentar para sua dimensão política. Nesse sentido, as palavras de Mezzadra e Neilson (2014, p. 15) são eloquentes: "A questão da fronteira como método não é simplesmente metodológica. É, antes de tudo, uma questão política [...] pode-se dizer que o método tem mais a ver com o agir sobre o mundo do que com o conhecê-lo". Nessa ótica, a abordagem do borderscaping oferece a oportunidade de repensar os imaginários mediterrâneos e a conexão migrações-fronteiras, praticando aquilo que Herzfeld (2001) definiu como "militant middle ground", ou seja, um terreno fértil de conexões e intersecções que está in-between, atravessando as teorias acadêmicas e a pesquisa aplicada, os desenhos globais e as histórias regionais e locais, os limites político-territoriais, que dizem respeito ao exercício da soberania e das fronteiras como categorizações sociais internas, que dizem respeito às políticas de identidade (Fassin, 2011). Desse modo, o desafio é superar a visão monolítica e essencializada do Mediterrâneo como o limite meridional da Fortaleza Europa ${ }^{10}$, revelando a historicidade das imaginações e das práticas de criação das fronteiras que estão subjacentes aos processos de b/ordering-othering (van Houtoum et al., 2005) no espaço-fronteira do Mediterrâneo através de uma abordagem metodológica politica e também performativo, como expressão da capacidade da perspectiva do borderscaping de emendar a lacuna entre representações e práticas. Isto permitiria repensar a conexão fronteira-migração no Mediterrâneo, contribuindo não só para uma

9 Em inglês no original italiano, traduzido como "campo intermédio militante". (N.T.).

10 "Fortaleza Europa" (em alemão: Festung Europa) é um termo originalmente usado pela propaganda militar de ambos os lados durante a II Guerra Mundial, referindo-se às áreas ocupadas pela Alemanha Nazista na Europa Continental, em oposição ao Reino Unido do outro lado do canal da Mancha. Atualmente, o termo é usado para contestar os regimes de fronteira e migração europeus. Pode se referir tanto às atitudes em relação aos migrantes, quanto às políticas de fortificação de fronteiras levadas a cabo, por exemplo, nos enclaves espanhois na África setentrional (Ceuta e Melilla) e no sistema de patrulhas fronteiriças e detenções empregadas com o objetivo de coibir a imigração ilegal para a União Europeia (Jünemann A., Fromm N., and Scherer N. (Eds.), 2017). 
renovada transformação crítica no conhecimento das fronteiras, mas também desenvolvendo ferramentas analíticas e operacionais para descrever as novas formas de pertença e devir que é urgente compreender e 'acolher' em suas complexidades, em vez de continuar a negar, excluir ou 'excluir-incluindo', em uma era de globalização e fluxos transnacionais (Anteby-Yemini et al., 2014).

\section{No borderscape ítalo-tunisiano: in-between Mazara e Mahdia}

As considerações apresentadas nos parágrafos anteriores sobre o potencial conceitual e metodológico da abordagem do borderscaping encontraram aplicação nas diretrizes adotadas para a pesquisa etnográfica realizada na região da fronteira ítalo-tunisiana com particular atenção a dois espaços urbanos - o de Mazara del Vallo na Sicília e o de Mahdia na Tunísia - lidos à luz de suas relações mútuas.

A cidade de Mazara del Vallo - na província de Trapani, no sudoeste da Sicília - está frente à África, 137 quilômetros através do Mar Mediterrâneo, estando geograficamente mais próxima da cidade de Túnis do que da bota italiana. Mazara não está apenas no borderscaping italo/tunisiano, sendo, portanto, uma cidade de limite, mas que se configura como um borderscape por si mesmo, uma cidade-limite, o que reflete a complexidade da região de fronteira em que está localizada (Cole, 2003). A cidade foi destino de um primeiro grande fluxo migratório de tunisianos para a Itália, ainda na década de setenta do século passado. No entanto, o antropólogo mazarense Cusumano (1976) descreveu bem esse fluxo de migrantes tunisianos para a Sicília e, em particular para Mazara, como um "retorno", para revelar como os fluxos migratórios contemporâneos entre a Tunísia e a Sicília são na verdade o epifenômeno dos grandes movimentos de homens e mercadorias através do Mediterrâneo ao longo dos séculos. Em particular, Cusumano fala de "retorno" no caso dos migrantes tunisianos em Mazara, referindo-se ao fato de que foi justamente desembarcando no porto de Mazara e partindo desta cidade que os árabes iniciaram a conquista da Sicília em 827 d.C., deixando sinais visíveis de sua presença na paisagem urbana de Mazara, entre as quais se destaca o centro histórico construído pelos árabes durante sua ocupação histórica e ainda chamado de casbah. Mazara tem uma relação particular com 
a cidade costeira tunisiana de Mahdia, local de origem da maioria dos imigrantes que vieram para Mazara ${ }^{11}$ ao longo dos anos. Localizada ao sul de Monastir e a sudeste de Sousse, Mahdia é a capital da delegação [espécie de distrito ou grande município, N.T.] e do gouvernorat [província, N.T.] homônimos, e um importante centro nacional e internacional para a indústria pesqueira, bem como para a indústria têxtil. Antes de ser um local de emigração para Mazara, Mahdia foi destino de muitos mazarenses que ali trabalharam, principalmente, na pesca, mas também em outras atividades industriais, até às décadas de 1940 e $1950^{12}$.

A atenção prestada à história da relação entre o borderscape urbano de Mazara e aquele de Mahdia relata um processo de progressiva "mediterranização" do espaço de fronteira no Canal da Sicília, através da qual a complexidade deste borderscape euro/africana encontra expressão (Ben-Yehoyada, 2011). Esta complexidade pode ser entendida através da representação multidimensional da fronteira que a abordagem do borderscaping permite, revelando como a fronteira ítalo/tunisiana não pode ser reduzida a uma linha estática demarcando os limites territoriais da autoridade e jurisdição política dos dois Estados envolvidos, como a sua representação cartográfica o impôs. Em vez disso, as lentes analíticas do borderscaping mostram que a fronteira não é uma geometria, mas um lugar complexo, móvel (no espaço e no tempo) e relacional, um espaço fluido atravessado por uma multiplicidade de negociações, reivindicações e contrarreivindicações socioculturais, políticas e econômicas, que se atualizam no nível das práticas cotidianas.

Diante disso, a reflexão conceitual e a pesquisa empírica se questionaram se a definição de formas virtuosas de 'humanizar' a fronteira, para aprender a ouvir e, ao mesmo tempo, dar voz à

11 É interessante notar que a cidade de Mazara é geminada com a de Mahdia desde 1973 e a geminação foi relançada e ampliada em 1991. As duas administrações têm colaborado em vários projetos de cooperação ao longo dos anos.

12 A imigração tunisiana para Mazara reflete um movimento maciço de emigração na direção oposta, da Sicília e do sul da Itália para a Tunísia, que, embora substancialmente ainda pouco conhecida, se estabeleceu na segunda metade do século XVIII e perdurou até os anos sessenta do século XX. Para mais informações, consultar Tartamella (2011). 
pluralidade de diferentes experiências que habitam e atravessam a região de fronteira ítalo/tunisiana, buscando também estratégias úteis para tornar visíveis essas experiências plurais. A experiência que se faz das fronteiras não é, de fato, igual para todos. Nessa perspectiva, a pesquisa se questionou em relação à elaboração de ferramentas conceituais e metodológicas úteis para descrever como as experiências da fronteira ítalo/tunisiana frequentemente se chocam com os pressupostos da teoria geopolítica e representações dos meios de comunicação de massa dominantes, mostrando como a retórica e as políticas das fronteiras são experienciadas, vividas e interpretadas por quem vive na fronteira. $\mathrm{O}$ valor da fronteira surge como um espaço político e social dinâmico e conflituoso, cuja gestão requer políticas capazes de restaurar a profundidade histórica e antropológica da fronteira - para além da linha - atentando para as condições contextuais específicas da fronteira como composta e continuamente recomposta na interação entre uma pluralidade de atores (não apenas os Estados e os atores institucionais), de discursos, práticas, normas e interesses (Brambilla, 2015a; 2015b).

\section{Jovens migrantes e experimentos metodológicos: para uma nova agência}

Entre os vários atores envolvidos na pesquisa, existe um grupo sobre o qual o estudo oferece um material particularmente significativo para reflexão. Trata-se de jovens migrantes de origem tunisiana que nasceram ou vivem em Mazara, cujas famílias são originárias de Mahdia ${ }^{13}$. Os jovens são geralmente excluídos da vida administrativa e política, forçados a uma cidadania 'diferida' ou 'reduzida', em alguns aspectos comparável à dos migrantes. Ouvir e dar voz às formas como esses jovens entendem o borderscaping que habitam significa reconhecer seu direito de participação na esfera pública,

$13 \mathrm{O}$ trabalho realizado com jovens migrantes tunisianos foi incorporado a um trabalho etnográfico mais amplo que utilizou diferentes métodos de pesquisa etnográfica (observação não-participante, diário etnográfico, entrevistas narrativas e semiestruturadas, grupos focais, fotografia e vídeo) e foi realizado entre a primavera de 2013 e o outono de 2015 em Mazara e em Mahdia, envolvendo vários atores adultos institucionais e não-institucionais em ambos os lados da fronteira. 
acolhendo sua agência como forma de resistência às representações e às práticas hegemônicas que se baseiam em uma concepção externalista e contemplativa, 'in-ativa', do borderscape ítalo/tunisiano. Significa também permitir atualizar-se do que de Certeau (2001, p. 7-16) definiu como "contramicrofísica das práticas cotidianas", realizada pelos jovens, que as inscrevem no borderscape em que habitam como espaço de vida e habitabilidade questionando configurações hegemônicas e buscando outras novas e "comuns".

Portanto, trabalhar com os jovens parece ser uma primeira condição importante para que novas formas de agência possam atualizar-se no Mediterrâneo. Envolver os jovens filhos de migrantes, tornar visível a complexidade 'viva' de suas subjetividades políticas, oferece oportunidades virtuosas para remediar as "patologias de in/ visibilidade" (Borren 2008, p. 219-225) sobre as quais se sustentam o espetáculo dos limites no Mediterrâneo, chamando a atenção, ao contrário, para as complexas práticas subjacentes à esfera pública diaspórica, na qual os migrantes podem ser agentes ativos de uma imagem diferente das migrações através do Mediterrâneo.

Coerentemente com essas considerações, o estudo com jovens mazarenses de origens tunisianas utilizou uma metodologia de pesquisa qualitativa interdisciplinar entre ciências sociais e humanas. Mais precisamente, métodos de narrativa visual (desenhos, autofotografia, fotoelicitação, mapas participativos e vídeos curtos) foram utilizados, bem como outros métodos de pesquisa qualitativa, incluindo caminhadas e o shadowing ${ }^{14}$ (sombreamento), de modo a incentivar capacidade dos jovens de se expressarem por meio de formas narrativas visuais, que chamam a atenção para a relevância de seu imaginário espacial, nos contando sobre sua reação com o borderscape que habitam ${ }^{15}$. O método videográfico teve

14 Shadowing é uma observação em movimento, uma forma móvel de observação não-participante. $O$ foco central dessa metodologia está em estabelecer dados detalhados e ricos sobre sujeitos específicos e suas ações no contexto em que são acompanhados por um determinado tempo. Os pesquisadores seguem os sujeitos em seus deslocamentos espaciais e sociais cotidianos. Para mais detalhes, consulte Czarniawska (2007).

15 Para uma análise mais detalhada do uso de métodos visuais, no estudo de um borderscape urbano, no que diz respeito aos imaginários e às experiências de crianças migrantes, consultar Brambilla (2015c). 
particular importância. As atividades com os jovens foram filmadas e incluídas no documentário "Houdoud al bahr, As fronteiras do mar: Mazara-Mahdia” e os próprios jovens gravaram parte das imagens ${ }^{16}$. Deste ponto de vista, o método videográfico e os outros métodos visuais utilizados são entendidos não somente como instrumentos estéticos, mas também analíticos e comunicativos, úteis não apenas para descrever visualmente discursos e práticas sociais, mas também para promover uma melhor compreensão das formas através das quais o borderscape é construído, percebido, interpretado e representado por quem nele vive. Seguindo a abordagem metodológica descrita, foram realizadas duas oficinas temáticas com os jovens: uma sobre o tema "A paisagem como mediador intercultural" e a outra sobre "A fronteira ítalo/tunisiana: imaginações, imaginários e imagens" ${ }^{17}$. Os métodos utilizados e a particular declinação temática das oficinas visavam estimular os jovens a problematizar sua relação com o espaço de fronteira que habitam, repensando-o não mais como uma geometria linear divisiva, mas como um espaço construído, habitado e reticular, como um espaço antropolítico em contínua evolução e, portanto, um espaço de oportunidades políticas, em cuja definição os jovens

$16 \mathrm{Na}$ sua versão integral e curta, o documentário (2015, idealização: Chiara Brambilla, direção: Chiara Brambilla e Sergio Visinoni, narrador e textos: Antonino Cusumano, pesquisa e consultoria antropológica: Chiara Brambilla e Alessio Angelo) pode ser visualizado online no link: http://www.cercounibg.it/hoududalbahr/.

17 As oficinas foram realizadas várias vezes durante 2014 e 2015. Em Mazara del Vallo, as oficinas foram realizadas com quatro grupos de jovens: 7-9, 9-10, 11-12, 16-19 anos. Com este último grupo, as atividades da oficina também foram realizadas em Mahdia durante os meses de verão, quando os adolescentes retornam à Tunísia para aproveitar as férias escolares. Os materiais iconográficos elaborados durante as atividades de oficina realizadas com os jovens foram reunidas e apresentados na exposição "Houdoud al Bahr|As fronteiras do mar" (Bérgamo, 1-5 de julho de 2016, idealização: Chiara Brambilla e Rita Ceresoli). Além dos materiais iconográficos elaborados durante as oficinas, a exposição inclui a série fotográfica "Notas sobre o borderscape ítalo-tunisiano" de Alessio Angelo (antropólogo e colaborador na coleta de dados do Projeto EUBORDERSCAPES para o estudo de caso da fronteira ítalo/tunisiana) e o documentário homônimo na exposição filmada no contexto da pesquisa. 
migrantes participam por meio de sua presença nas geografias cotidianas do borderscape ítalo-tunisiano.

\section{Habitar o borderscape ítalo-tunisiano: rumo a uma cidadania de inspiração mediterrânea}

A pesquisa realizada na área da fronteira ítalo/tunisiana revela uma agência que é gerada no habitar o borderscape por parte dos jovens mazarenses de origem tunisiana. Trata-se de uma agência que se configura como possibilidade de ação e participação política dos jovens migrantes tunisianos a partir de sua residência naquele local em particular. É vivendo no borderscape ítalo/tunisiano "entre" Mazara e Mahdia, vivendo naquele in-between, que novas formas de agências se atualizam.

Através de suas trajetórias de vida espaço-social, suas múltiplas percepções, experiências e imaginações do borderscape ítalo/ tunisiano, os jovens mazarenses de origens tunisianas se recusam a ter que escolher entre ser italianos, tunisianos ou globais. Esses jovens 'encarnam' o borderscape e praticam uma identidade que tira sua forma específica e seu caráter flexível do serem habitantes da "constelação mediterrânea" que se desenvolveu no Canal da Sicília ao longo dos séculos (Ben-Yehoyada 2011, p. 388-390).

Nesse contexto, a agência dos jovens reflete um fluxo de identidade transnacional que encontra expressão em múltiplos sentidos de pertencimento ao lugar, os quais descrevem um apego emocional a ele, mais do que uma reivindicação de cidadania legal (Brambilla, 2016). Esta agência faz desses jovens migrantes tunisianos os precursores e os criadores de uma nova e original forma de cidadania, uma cidadania de inspiração mediterrânea, baseada na estreita ligação, evadida das geografias políticas estadocêntricas modernas, entre a dimensão política e a antropológica e emocional. Os imaginários e as experiências do borderscape dos jovens migrantes tunisianos podem ser considerados como formas de resistência na vida cotidiana aos imaginários e às imagens dominantes que essencializam o Mediterrâneo no limite sul da fortaleza Europa. Os jovens oferecem uma 'contraimagem' do borderscape ítalo/tunisiano, através de uma resistência que não se concretiza com uma oposição explícita 
às categorizações modernas de pertencimento político, mas que se origina na presença política cotidiana dos jovens no espaço-fronteira mediterrâneo, abrindo-se à possibilidade de novas formas de pertencimento político, não mais entendido como um status adquirido ou a ser adquirido, mas como um movimento animado por atos e práticas.

Os imaginários alternativos da fronteira mediterrânea, que dela emergem, nos convidam a repensar o Mediterrâneo, abandonando a retórica dos meios de comunicação que querem que seja palco da crise dos refugiados e dos migrantes, e descrevendo-o, ao invés, como um espaço de criatividade política, onde se torna possível elaborar uma "política da esperança", ou seja, uma política das possibilidades que estão por vir (Appadurai, 2014, p. 297412). Os jovens migrantes tunisianos que vivem 'entre' Mazara e Mahdia, 'encarnando' o borderscape mediterrâneo em que vivem, oferecem um exemplo significativo desta política da esperança, que encontra expressão na ideia de uma cidadania de inspiração mediterrânea. Prenunciar tal política da esperança significa rejeitar completamente a violência e as imposições dos regimes fronteiriços e os empecilhos à busca do exercício dessa cidadania. É necessário, ao invés, e, inevitavelmente, aprender a olhar para os espaços onde novas formas de agência política se originam, revelando - como Aitken (2014) bem descreve em seu volume sobre a etnopoética do espaço e o potencial transformativo virtuoso da agência dos jovens - um espaço de possibilidades para 'horizontes' de esperança sem precedentes, através dos quais se afirma um novo imaginário da conexão fronteiras-migrações no Mediterrâneo, para além da linha do porvir.

Deste ponto de vista, os borderscapes do Mediterrâneo são lugares importantes a considerar a fim de redescobrir, como argumenta Fassin (2010, p. 23), uma política que, lembrando a reflexão arendtiana, não se baseie nas armadilhas territoriais da identidade nacional, mas sim na pluralidade humana como condição substantiva à toda vida política; uma política que se baseia na reciprocidade do ser diferente e assume a diferença como princípio através do qual se constrói uma igualdade potencial e futura, mas não utópica. 


\section{Referências}

AITKEN, S. The Ethnopoetics of Space and Transformation: Young People's Engagement, Activism and Aesthetics. Farnham: Ashgate, 2014 ANTEBY-YEMINI, L.; BABY-COLLIN, V.; MAZZELLA, S.; MOURLANE, S.; PARIZOT, C.; REGNARD, C.; SINTÈS, P. (a cura di). Borders, Mobilities and Migrations. Perspectives from the Mediterranean, 19-2 $1^{\text {st }}$ Century. Bruxelles: Peter Lang, 2014

APPADURAI, A. Il futuro come fatto culturale. Saggi sulla condizione globale. Milano: Cortina, 2014

BECHEV, D.; NICOLAIDIS, K. (a cura di). Mediterranean Frontiers. Borders, Conflict and Memory in a Transnational World. Londra - New York: Tauris Academic Studies, 2010

BEN-YEHOYADA, N. The Moral Perils of Mediterraneanism: Second- Generation Immigrants Practicing Personhood between Sicily and Tunisia. Journal of Modern Italian Studies, v. 16, n. 3, 2011, p. 386-403.

BORREN, M. Towards an Arendtian Politics of In/visibility: On Stateless Refugees and Undocumented Aliens. Ethical Perspectives: Journal of the European Ethics Network, v. 15, n. 2, 2008, p. 213-237.

BRAMBILLA, C. Borders Still Exist! What Are Borders?. In: RICCIO, B.; BRAMBILLA, C. (a cura di). Transnational Migration, Cosmopolitanism and Dis- located Borders. Rimini: Guaraldi, 2010, p. 73-85,

BRAMBILLA, C. Shifting Italy/Libya Borderscapes at the Interface of EU/ Africa Borderland: A "Genealogical” Outlook from the Colonial Era to Post-colonial Scenarios. ACME An International E-journal for Critical Geographies, v. 13, n. 2, 2014, p. 220-245.

BRAMBILLA, C. Exploring the Critical Potential of the Borderscapes Concept. Geopolitics, v. 20, n. 1, 2015a, p. 14-34.

BRAMBILLA, C. Navigating the Euro/African Border and Migration Nexus through the Borderscapes Lens: Insights from the LampedusaInFestival. In: BRAMBILLA, C.; LAINE, J.; SCOTT, J.W.; BOCCHI, G. (a cura di), Borderscaping: Imaginations and Practices of Border Making. Farnham: Ashgate, 2015b, p. 111-121.BRAMBILLA, C. Mobile Euro/African Borderscapes: Migrant Communities and Shifting Urban Margins In: AMILHAT SZARY, A.-L.; GIRAUT, F. (a cura di). Borderities and the Politics of the Contemporary Mobile Borders. Basingstoke - Londra: Palgrave McMillan, 2015c, p. 138-154.

BRAMBILLA C. Borderscaping: Estetica | Politica | Trans-territorialità. 
Nuove agency geografico-politiche nel Mediterraneo "oltre la linea". Semestrale di Studi e Ricerche di Geografia, XXVIII(1), 2016, p. 77-90. BRAMBILLA C., LAINE J., SCOTT J.W., BOCCHI G. (a cura di). Borderscaping: Imaginations and Practices of Border Making. Farnham: Ashgate, 2015

BRAUDEL, F. Il Mediterraneo. Lo spazio, la storia, gli uomini, le tradizioni. Milano: Bompiani, 1987CHAMBERS, I. Mediterranean Crossings. The Politics of an Interrupted Modernity. Durham - Londra: Duke University Press, 2008

COLE, J.E. Borders Past and Present in Mazara del Vallo, Sicily. European Studies: A Journal of European Culture, History and Politics, v. 19, 2003, p. 195-216.

CUSUMANO, A. Il ritorno infelice. I tunisini in Sicilia. Palermo: Sellerio, 1976.

CUTTITTA, P. Lo spettacolo del confine. Lampedusa tra produzione e messa in scena della frontiera. Sesto San Giovanni: Mimesis, 2012

CZARNIAWSKA, B. Shadowing and Other Techniques for Doing Fieldwork in Modern Societies. Liber: Copenhagen Business School Press, 2007

DE CERTEAU, M. L'invenzione del quotidiano. Roma: Edizioni Lavoro, 2001.

DE GENOVA, N. Spectacles of Migrant "Illegality": The Scene of Exclusion, the Obscene of Inclusion. Ethnic and Racial Studies, v. 36, .n. 7, 2013, p. 1180-1198.

DELL'AGNESE, E.; AMILHAT-SZARY, A.-L. Introduction. Borderscapes: From Border Landscapes to Border Aesthetics. Geopolitics, v. 20, n. 1, 2015, p. 4-13.

FASSIN, D. (a cura di). Les nouvelles frontiers de la société française. Parigi: Éditions La Découverte, 2010.

FASSIN, D. Policing Borders, Producing Boundaries. The Governmentality of Immigration in Dark Times. Annual Review of Anthropology, v. 40, 2011, p. 213-226.

HERZFELD, M. Anthropology: Theoretical Practice in Culture and Society. Malden: Wiley-Blackwell, 2001

HESS, S.; KASPAREK, B. (a cura di). Grenzregime. Diskurse, Praktiken, Institutionen in Europa, Berlino - Amburgo, Assoziation A, 2010. 
JÜNEMANN, A.; FROMM, N.; SCHERER, N. (Eds.). Fortress Europe? Challenges and Failures of Migration and Asylum Policies. Wiesbaden: Springer, 2017.

MEZZADRA, S.; NEILSON, B. Confini e frontiere. La moltiplicazione del lavoro nel mondo globale. Bologna: Il Mulino, 2014.

MORIN, E. Introduzione a una politica dell'uomo. Roma: Meltemi, 2010 OLWIG, K. Performing on the Landscape versus Doing Landscape: Preambulatory Practice, Sight and the Sense of Belonging. In: INGOLD, T.; VERGUNST, J.L. (a cura di) Ways of Walking. Ethnography and Practice on Foot. Aldershot: Ashgate, 2008, p. 81-91.

PARKER N.; VAUGHAN-WILLIAMS, N. Critical Border Studies: Broadening and Deepening the "Lines in the Sand" Agenda. Geopolitics, v. 17, n. 4, 2012, p. 727- 733.

RAJARAM, P.K. Beyond Crisis: Rethinking the Population Movements at Europe's Border. FocaalBlog, 2015. Disponível em: www.focaal-blog. com/2015/10/19/prem-kumar-rajaram-beyond-crisis.

RAJARAM, P.K.; GRUNDY-WARR, C. Introduction. In: RAJARAM, P.K.; GRUNDY- WARR, C. (a cura di).Borderscapes: Hidden Geographies and Politics at Territory's Edge. Minneapolis: University of Minnesota Press, 2007, p. ix-xl.

RANCIÈRE, J. Il disaccordo. Politica e filosofia. Roma: Meltemi, 2007.

RANCIÈRE, J. Estetica e politica. Dialogo sulla partizione del sensibile. Roma: Edizioni Alegre, 2011

RUMFORD, C. Guest Editorial on Global Borders. An Introduction to the Special Issue, Environment and Planning D. Society and Space, v. 28, n. 6, 2010, p. 951-956.

SCHIMANSKI, J.; WOLFE, S. The Aesthetics of Borders. In: AUKRUST, K. (a cura di), Assigning Cultural Values. Francoforte sul Meno: Peter Lang, 2013, p. 235-250.

SCHIMANSKI, J.; WOLFE, S. (a cura di). Border Aesthetics. Concepts and Intersections. New York - Oxford: Berghahn Books, 2017.

TARTAMELLA, E. Emigranti anomali. Italiani in Tunisia tra Otto e Novecento. Trapani: Maroda: Editori, 2011.

VAN HOUTUM, H.; KRAMSCH, O.; ZIERHOFER, W. (a cura di). B/Ordering Space. Aldershot: Ashgate, 2005.

WILSON, T.; DONNAN, H. (a cura di). A Companion to Border Studies. Chichester: Blackwell Publishing, 2012. 